\title{
La estructura de las comunidades de helmintos de Galaxias maculatus (Osmeriformes: Galaxiidae) en diferentes sitios de un lago de la Patagonia argentina
}

\author{
María V. Fernández ${ }^{1, \bigotimes}$; Liliana Semenas ${ }^{2} \&$ Gustavo Viozzi $^{2}$ \\ ${ }^{1}$ Laboratorio de Gestión y Manejo de Recursos Ícticos, INIBIOMA (CONICET-Universidad Nacional del Comahue). \\ QUINTRAL 1250. San Carlos de Bariloche.²aboratorio de Parasitología, INIBIOMA (CONICET-Universidad \\ Nacional del Comahue). Quintral 1250, (8400) Bariloche, Argentina.
}

\begin{abstract}
RESUMEN. La variación espacial de las comunidades de helmintos en un sistema acuático puede atribuirse a diferentes factores bióticos y abióticos. En América del Sur, este tipo de estudios no ha sido realizado en ambientes de agua dulce. El lago Nahuel Huapi es el de mayor tamaño dentro del Parque Nacional homónimo; presenta una gran variedad de hábitats y constituye un ambiente ideal para identificar los factores que influyen en las variaciones de las comunidades de helmintos de Galaxias maculatus (puyen chico) en diferentes sitios del lago. Para analizar estas variaciones, en el lago se muestrearon cinco sitios con diferentes características ambientales y se examinaron, en total, 144 puyenes. Se realizaron análisis comunitarios y se relacionó la composición de las comunidades y la abundancia de las diferentes especies de helmintos con los factores bióticos y abióticos de los diferentes sitios. El tamaño del hospedador, las zonas litorales someras con vegetación acuática y el impacto antrópico fueron los factores que determinaron las variaciones en las infracomunidades y en las comunidades componentes de helmintos que parasitan al pez presa Galaxias maculatus en los diferentes sitios del lago Nahuel Huapi.
\end{abstract}

[Palabras clave: parásitos, lago Nahuel Huapí, puyen chico]

\begin{abstract}
Aвstract. The helminth communities structure of Galaxias maculatus (Osmeriformes: Galaxiidae) in different sites of an Argentinean Patagonian lake. The spatial variation of helminth communities of an aquatic system can be attributed to different biotic and abiotic factors. In South America, this type of studies has not been conducted in freshwater environments. The lake Nahuel Huapi is the largest within the Nahuel Huapi National Park. As it presents a diversity of habitat, this lake offers an ideal environment to identify factors that impact over the helminth communities of Galaxias maculatus (small puyen) at different sites of the lake. In order to analyze these variations, 144 small puyens were captured in five sites of the lake with different environmental characteristics. Community analyses were performed, and composition of communities and abundance of the different species of helminths were related with biotic and abiotic factors of the different sites. The size of the host, shallow littorals with aquatic vegetation, and anthropic impact were the factors that determined the variations in composition and abundance of the component communities of helminths in the prey fish Galaxias maculatus from Nahuel Huapi Lake.
\end{abstract}

[Keywords: parasites, Nahuel Huapí lake, small puyen]

\section{INTRODUCCIÓN}

Tanto la composición de las comunidades de helmintos como la abundancia de las distintas especies de parásitos pueden proveer información sobre las características ambientales y la distribución espacial e interacciones tróficas de los hospedadores. Las variaciones en estas comunidades pueden atribuirse a la disponibilidad, la especificidad y el rango de distribución de los hospedadores intermediarios y definitivos (Wisniewski 1958; Karvonen \& Valtonen 2004; Ternengo et al. 2009). Las diferencias entre las comunidades componentes de helmintos de un pez pueden deberse a factores tales como su tamaño, su nivel trófico, las características ambientales y

\section{Editor asociado: Fernando Unrein}

valeria.fernandez@crub.uncoma.edu.ar la composición de la fauna acuática y terrestre del cuerpo de agua y sus alrededores (Fellis \& Esch 2005; Salgado-Maldonado et al. 2005; Lafferty 2008; Anderson \& Sukhdeo 2009; Fernández et al. 2010; Rodhe 2010; Timi et al. 2010). Por su parte, las similitudes pueden atribuirse, entre otros factores, al movimiento de aves y mamíferos piscívoros entre sitios (Hartvigsen \& Kennedy 1993; Fernández et al. 2010).

Las investigaciones sobre las comunidades de parásitos de una única especie de pez de agua dulce en ambientes de la misma cuenca son escasos y se pueden citar los estudios de Coregonus lavaretus en Finlandia (Karvonen \& Valtonen 2004), Cichlasoma urophtalmus,

Recibido: 16 de febrero de 2015, Fin de arbitraje: 9 de mayo de 2015, Última versión revisada: 16 de julio de 2015, Aceptado: 1 de septiembre de 2015. 
Heterandria bimaculata y Profundulus punctatus en México (Salgado-Maldonado \& Kennedy 1997; Vidal Martínez \& Poulin 2003; PinachoPinacho et al. 2014; Salgado-Maldonado et al. 2014), Rhodeus amarus en el sudeste de Europa (Dávidová et al. 2011) y Lepomis gibbosus en Canadá (Chapman et al. 2015), aunque sólo estos últimos autores hacen una relación entre la estructura de las comunidades de parásitos con factores ambientales. No existen en el mundo estudios de este tipo en diferentes sitios de un mismo lago. El lago Nahuel Huapi constituye un ambiente ideal para realizar un estudio de estas características dado su gran tamaño, la diversidad ambiental que presenta en todo su perímetro y la alta abundancia del pez presa Galaxias maculatus (puyen chico). En base a este escenario se trabajó bajo la hipótesis de que las diferencias en los factores ambientales bióticos y abióticos entre los distintos sectores de un gran lago se reflejan en la estructura de la comunidad componente y de las infracomunidades de helmintos que parasitan a G. maculatus en diferentes sitios del lago Nahuel Huapi.

\section{MATERIALES y MÉTOdos}

El lago Nahuel Huapi (Figura 1) está situado a 764 m.s.n.m. y a $41^{\circ} 04^{\prime} \mathrm{S}-71^{\circ} 25^{\prime}$ O. Pertenece a la cuenca del río Limay y es el lago natural más grande dentro del Parque Nacional Nahuel Huapi. Presenta forma extendida de oeste a este, con siete brazos. Posee una superficie de $557 \mathrm{~km}^{2}$, una profundidad máxima de $464 \mathrm{~m}$ y la longitud de sus costas es de 357 km (Quirós 1990; Pedrozo \& Vigliano 1995). El $85 \%$ de los días del año sopla viento del oeste. Las costas del oeste tienen poco efecto antrópico, pero hacia el sureste, donde se ubica la ciudad de San
Carlos de Bariloche, este efecto se incrementa por la descarga de efluentes domiciliarios. Por su parte, la costa suroeste presenta bajo efecto antrópico debido a la ausencia de asentamientos poblacionales y a la dificultad de acceso (Pedrozo et al. 1997). El sector oeste del lago Nahuel Huapi se caracteriza por su costa de relieve abrupto, con acantilados (principalmente en el Brazo Blest), entre los que se intercalan escasos sectores de playas pequeñas y angostas. El sector este presenta litorales someros con playas amplias constituidas por distintos materiales como rocas, cantos rodados y arena fina y gruesa (Tabla 1).

La vegetación acuática emergente está representada por los juncos Juncus sp. y Scirpus californicus, que aparecen en manchones en lugares someros. La fauna de peces está representada por 5 especies autóctonas (el bagre aterciopelado [Olivaichthys viedmensis], el puyen chico [Galaxias maculatus], el puyen grande [Galaxias platei], el pejerrey [Odontesthes hatcheri] y la perca [Percichthys trucha]) y 3 introducidas (la trucha arco iris [Oncorhynchus mykiss], la trucha marrón [Salmo trutta] y la trucha de arroyo [Salvelinus fontinalis]).

Galaxias maculatus es un pez presa de tamaño pequeño que se distribuye en casi toda la Patagonia. Domina numéricamente los ensambles de peces de la mayoría de los lagos donde habita.

Tabla 1. Características de los cinco sitios muestreados. Table 1. Characteristics of the five sampling sites

\begin{tabular}{lccccc}
\hline \multicolumn{1}{c}{ Sitio } & $\begin{array}{c}\text { Brazo } \\
\text { Machete }\end{array}$ & $\begin{array}{c}\text { Puerto } \\
\text { Blest }\end{array}$ & $\begin{array}{c}\text { Club } \\
\text { Regatas }\end{array}$ & $\begin{array}{c}\text { Muelle } \\
\text { Bariloche }\end{array}$ & $\begin{array}{c}\text { Ortiz } \\
\text { Basualdo }\end{array}$ \\
\hline $\begin{array}{l}\text { Impacto } \\
\text { antrópico }\end{array}$ & bajo & bajo & medio & alto & bajo \\
$\begin{array}{l}\text { Vegetación } \\
\text { acuática }\end{array}$ & escaso & abundante abundante & ausente & escaso \\
$\begin{array}{l}\text { Litoral } \\
\text { somero }\end{array}$ & angosto & angosto & amplio & amplio & amplio \\
$\begin{array}{l}\text { Exposición } \\
\text { predominante } \\
\text { del viento }\end{array}$ & $\sin$ & $\sin$ & $\sin$ & con & $\sin$ \\
\end{tabular}

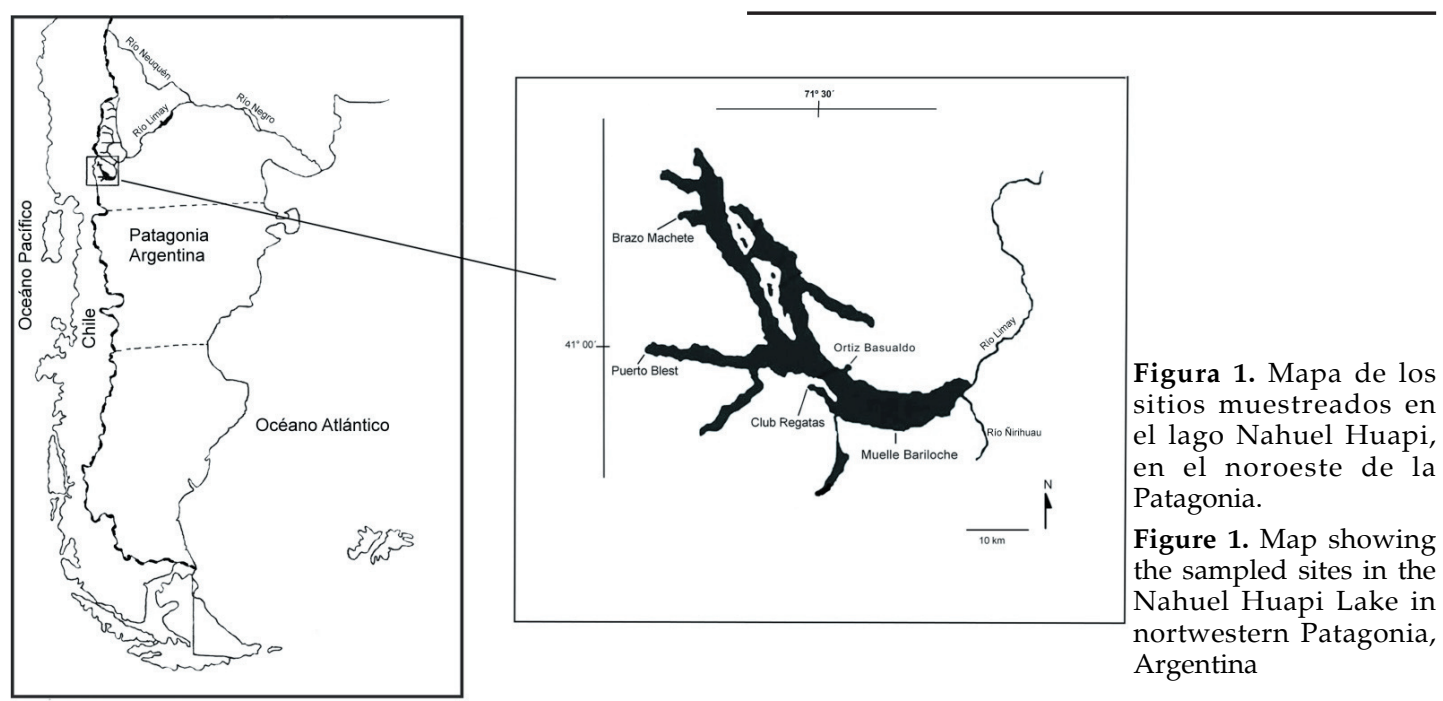


En el noroeste de la Patagonia argentina sólo se encuentran poblaciones encerradas que presentan migraciones estacionales para desovar en lugares someros con vegetación acuática. Las larvas eclosionan y migran a la zona pelágica, donde se alimentan de zooplancton (Barriga et al. 2012); seis meses después se produce la metamorfosis larval y retornan a los litorales donde nacieron (Cussac et al. 1992; Barriga et al. 2002; Macchi 2004; Rechencq et al. 2011; Carrea et al. 2012).

Se eligieron cinco sitios: Puerto Blest, Brazo Machete, Club Regatas, Ortiz Basualdo y Muelle Bariloche (Figura 1). Los mismos difieren en la combinación de las siguientes características: impacto antrópico, presencia de vegetación acuática, presencia de litorales someros y exposición al viento predominante (Tabla 1).

\section{Captura de peces y procesamiento de datos}

Durante la primavera de 2008 y el verano de 2009 se capturaron puyenes con redes de arrastre y nasas cebadas en los cinco sitios seleccionados del lago Nahuel Huapi. En cada sitio se muestrearon 30 puyenes, a excepción del sitio Puerto Blest, donde se capturaron 24. El tamaño de muestra fue calculado sobre la base de trabajos no publicados en los que, con muestras de 15 especímenes de diferentes lagos, se registraron entre $75 \%$ y $100 \%$ de las especies parásitas presentes en Galaxias maculatus. Los peces fueron trasladados vivos al laboratorio y mantenidos a temperatura controlada $\left(8^{\circ} \mathrm{C}\right)$ hasta su procesamiento. Se midió la longitud estándar y se realizó la disección bajo microscopio estereoscópico. Se les revisó: tegumento, aletas, branquias, cerebro, ojos, corazón, bazo, hígado, vesícula biliar, estómago, ciegos e intestino, cavidad abdominal, vejiga gaseosa, gónadas, riñón y ductos urinarios. Los helmintos fueron contados y se registró el sitio de infección. Los especímenes colectados se fijaron en alcohol $70 \%$ ó formol $2 \%$ para su identificación taxonómica posterior. Se registró la riqueza de la comunidad componente de helmintos (la comunidad de helmintos de cada sitio) y de las infracomunidades (comunidad de helmintos por pez) (Bush et al. 1997) y se calculó la dominancia de Berger-Parker para cada sitio. Además, se calcularon las abundancias, las prevalencias y las intensidades medias de cada especie (Bush et al. 1997) y se clasificaron a los ciclos parasitarios como alogénicos (ciclos cuyos hospedadores definitivos son animales terrestres) y autogénicos (ciclos cuyos hospedadores definitivos son animales acuáticos) (Esch et al. 1990).

Para caracterizar y comparar las comunidades de helmintos se utilizó la prueba de Kruskal-Wallis y comparaciones múltiples de Tukey; éste permitió analizar las riquezas de las infracomunidades de los diferentes sitios del lago. El índice de Bray Curtis permitió determinar la similitud entre las comunidades componentes a través de la abundancia de las distintas especies de helmintos. Los datos se graficaron mediante un escalamiento multidimensional (MDS). Para descartar que la proximidad geográfica fuera un factor que promueve la similitud entre comunidades componentes se realizó una prueba de Mantel, midiendo sobre imágenes de Google Earth la distancia en línea recta desde el punto de muestreo de un sitio hasta los puntos de muestreo de los restantes sitios.

Se utilizó un modelo lineal generalizado para analizar la influencia del tamaño de los puyenes y las características ambientales de los sitios sobre la composición y la abundancia de las comunidades de helmintos. Se utilizó el logaritmo natural de la abundancia de parásitos de cada una de las especies como variable respuesta y las variables predictoras fueron divididas en cualitativas (impacto antrópico, presencia de vegetación acuática, exposición al viento predominante y presencia de litoral somero) y cuantitativa (longitud estándar de los puyenes en cada sitio). Se clasificaron las variables cualitativas de la siguiente manera: a) el impacto antrópico se dividió en tres categorías contabilizando las construcciones sobre un área rectangular de $500 \mathrm{~m}$ de largo sobre la línea de costa con centro en el sitio de muestreo, por $200 \mathrm{~m}$ de ancho perpendiculares a esta línea; se consideró efecto antrópico bajo (entre 0 y 4 casas), medio (entre 5 y 11 casas) y alto (más de 11 casas); b) la vegetación acuática emergente se estimó mediante imágenes de Google Earth y se chequeó con observaciones en el campo. Se contabilizó su abundancia en una longitud de 500 m de línea de costa, con centro en el sitio de muestreo. La abundancia fue categorizada como ausente (sin vegetación acuática), escasa (hasta $25 \%$ de cobertura de la costa del sitio de muestreo), media (entre 25 y 50\% de cobertura) y abundante (más de $50 \%$ de la costa con vegetación acuática); c) el litoral se clasificó como amplio (entre 30 y 200 $\mathrm{m}$ de ancho) o angosto (entre 10 y $30 \mathrm{~m}$ de ancho); y d) la exposición a los vientos predominantes se clasificó en "con" y "sin" exposición.

\section{Resultados}

La longitud estándar de los peces varió entre 30.3 y $54.2 \mathrm{~mm}$, y el promedio por sitio varió entre 39.4 y 45.1 (Tabla 2). Todos los peces capturados fueron juveniles y adultos (según Barriga et al. [2012]). La fauna de helmintos de los 144 peces procesados de los cinco sitios del lago Nahuel Huapi tuvo una riqueza total de 15 especies y estuvo compuesta por 5 digeneos, 2 monogeneos, 2 cestodes, 1 acantocéfalo y 5 nematodes (Tabla 2). La riqueza de especies entre sitios varió entre 9 (Club Regatas) y 12 (Muelle Bariloche y Brazo Machete). La riqueza de las infracomunidades de todos 
Tabla 2. Largo estándar de Galaxias maculatus (rango y promedio), presencia y riqueza de helmintos.

Table 2. Galaxias maculatus standard length (range and average), presence and richness of helminths.

\begin{tabular}{|c|c|c|c|c|c|c|}
\hline \multirow{3}{*}{$\begin{array}{l}\text { Sitio } \\
\text { G. maculatus } \\
\text { largo estándar }(\mathrm{mm})\end{array}$} & & \multirow{2}{*}{$\begin{array}{c}\text { Brazo Machete } \\
35-53.4\end{array}$} & \multirow{2}{*}{$\begin{array}{c}\text { Puerto Blest } \\
30.3-53.2\end{array}$} & \multirow{2}{*}{$\begin{array}{c}\text { Club Regatas } \\
33.3-49.8\end{array}$} & \multicolumn{2}{|c|}{ Muelle Bariloche Ortiz Basualdo } \\
\hline & Rango & & & & $33.3-51.1$ & $34.2-54.2$ \\
\hline & Media & 42 & 45.1 & 39.4 & 39.6 & 43.2 \\
\hline Tylodelphys sp. $(\mathrm{L})^{*}$ & & $\mathrm{x}$ & $\mathrm{x}$ & $x$ & $x$ & $\mathrm{x}$ \\
\hline \multicolumn{2}{|c|}{ Posthodiplostomum sp. $(\mathrm{L})^{*}$} & & $\mathrm{x}$ & $\mathrm{x}$ & & \\
\hline \multicolumn{2}{|c|}{ Steganoderma szidati (A) } & $\mathrm{x}$ & $\mathrm{x}$ & & $\mathrm{x}$ & $\mathrm{x}$ \\
\hline \multicolumn{2}{|c|}{ Stephanoprora uruguayense $(\mathrm{L})^{*}$} & $\mathrm{x}$ & $\mathrm{x}$ & $\mathrm{x}$ & $\mathrm{x}$ & $\mathrm{x}$ \\
\hline \multicolumn{2}{|c|}{ Acanthostomoides apophalliformis (L) } & $\mathrm{x}$ & $\mathrm{x}$ & $\mathrm{x}$ & $\mathrm{x}$ & $\mathrm{x}$ \\
\hline \multicolumn{2}{|c|}{ Philureter trigoniopsis (A) } & $\mathrm{x}$ & & $\mathrm{x}$ & $\mathrm{x}$ & $\mathrm{x}$ \\
\hline \multicolumn{2}{|l|}{ Gyrodactylus sp. (A) } & $\mathrm{x}$ & & & & $x$ \\
\hline \multicolumn{2}{|c|}{ Diphyllobothrium sp. (L)* } & & & & $\mathrm{x}$ & $\mathrm{x}$ \\
\hline \multicolumn{2}{|l|}{ Ailinella mirabilis $(\mathrm{L})$} & $\mathrm{x}$ & & $\mathrm{x}$ & $\mathrm{x}$ & \\
\hline \multicolumn{2}{|c|}{ Acanthocephalus tumescens (A) } & $\mathrm{x}$ & $\mathrm{x}$ & & $\mathrm{x}$ & \\
\hline \multicolumn{2}{|l|}{ Contracaecum sp. $(\mathrm{L})^{*}$} & $\mathrm{x}$ & $\mathrm{x}$ & & $\mathrm{x}$ & $\mathrm{x}$ \\
\hline \multicolumn{2}{|c|}{ Hysterothylacium patagonense $(\mathrm{L})$} & $\mathrm{x}$ & $\mathrm{x}$ & $\mathrm{x}$ & $\mathrm{x}$ & $\mathrm{x}$ \\
\hline \multicolumn{2}{|c|}{ Placonema pataguense (A) } & & $\mathrm{x}$ & & & \\
\hline \multicolumn{2}{|l|}{ Camallanus corderoi $(\mathrm{L})$} & $\mathrm{x}$ & $\mathrm{x}$ & $\mathrm{x}$ & $\mathrm{x}$ & $\mathrm{x}$ \\
\hline \multicolumn{2}{|l|}{ Hedruris suttonae (A) } & $\mathrm{x}$ & $\mathrm{x}$ & $\mathrm{x}$ & $\mathrm{x}$ & $\mathrm{x}$ \\
\hline \multicolumn{2}{|l|}{ Riqueza } & 12 & 11 & 9 & 12 & 11 \\
\hline \multicolumn{7}{|c|}{$\mathrm{L}=$ larva; $\mathrm{A}=$ adulto. ${ }^{*}=$ especies alogénicas } \\
\hline
\end{tabular}

L=larva; A=adulto. * =especies alogénicas

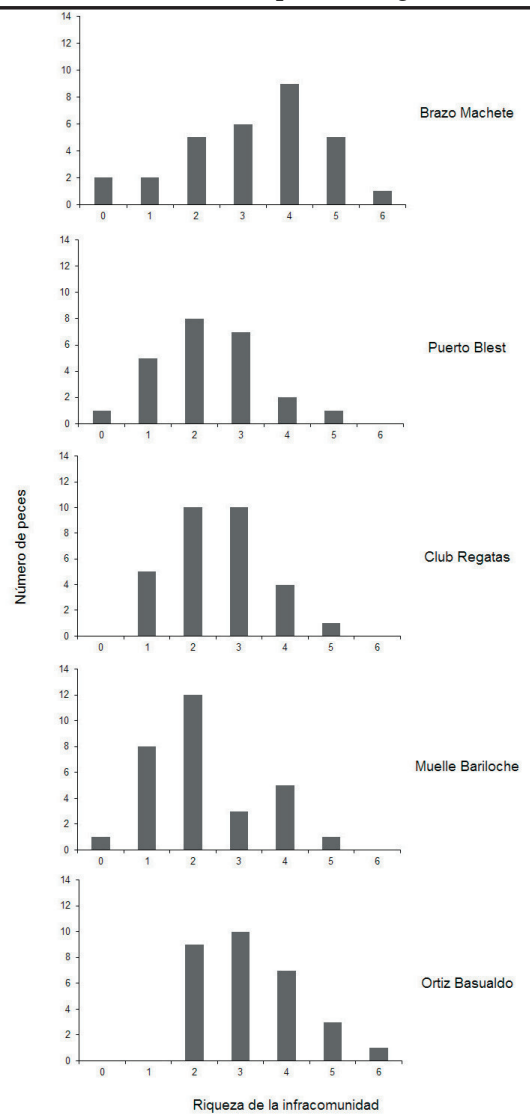

Figura 2. Riqueza de la infracomunidad de helmintos de Galaxias maculatus en los cinco sitios del lago Nahuel Huapi.

Figure 2. Infracomunity richness of Galaxias maculatus helminth parasite in five sites of Lake Nahuel Huapi.

los sitios muestreados presentó un rango de 0 a 6. El mayor rango de variación se registró en Brazo Machete (0-6) y el menor, en Ortiz Basualdo (2-6) y Club Regatas (1-5) (Figura 2). Se encontraron diferencias significativas de la riqueza de las infracomunidades entre los diferentes sitios estudiados (KW: $\mathrm{H}=18.27601$; $\mathrm{N}=144 ; P=0.0011$ ). La prueba de Tukey de comparaciones múltiples de la riqueza de infracomunidades de los cinco sitios indicó diferencias significativas entre el Muelle Bariloche y Ortiz Basualdo y entre el Muelle Bariloche y Brazo Machete (Tabla 3).

Se recuperó un total de 14779 helmintos. El sitio Ortiz Basualdo presentó la mayor abundancia, con un total de 6771 helmintos, de los cuales 6572 fueron especímenes de Tylodelphys sp. y 120 de Stephanoprora uruguayense. La menor abundancia total fue registrada en el sitio Muelle Bariloche (1103). La similitud de las comunidades componentes basada en la abundancia de las especies no está correlacionada con la distancia entre los sitios (prueba de Mantel: $\mathrm{rM}=-0.1317$; $P=0.534$ ). La representación gráfica del índice de Bray Curtis mediante un escalamiento multidimensional (MDS), con un valor de estrés de 0.2 , mostró un primer grupo formado por los sitios Brazo Machete, Puerto Blest y Club Regatas, ubicados en la parte superior del diagrama, y un segundo grupo formado por 
los sitios Muelle Bariloche y Ortiz Basualdo, ubicados en la parte inferior del diagrama (Figura 3). El modelo lineal generalizado indica que la abundancia de las diferentes especies en las comunidades de helmintos de G. maculatus está asociada al tamaño del pez, al impacto antrópico, a la presencia de litoral somero y a la vegetación acuática emergente en las costas. El viento fue el único factor que no mostró asociación con las abundancias parasitarias (Tabla 4).

La prevalencia más alta $(96 \%)$ se registró para Tylodelphys sp. en Puerto Blest, mientras que la intensidad media más alta (231) se registró para la misma especie en Ortiz Basualdo (Tabla 5). La especie dominante fue Tylodelphys sp., con valores de dominancia entre 0.90 y 0.99 en todos los sitios (Tabla 6).

Tabla 3. Comparación mútiple prueba de Tukey de la riqueza de las infracomunidades de helmintos en los cinco sitios del lago Nahuel Huapi.

Table 3. Multiple comparisons Tukey test of the infracommunity richness from the five sites from Nahuel Huapi Lake.

\begin{tabular}{lccccc}
\hline & $\begin{array}{c}\text { Brazo } \\
\text { Machete }\end{array}$ & $\begin{array}{c}\text { Brazo } \\
\text { Blest }\end{array}$ & $\begin{array}{c}\text { Club } \\
\text { Regatas }\end{array}$ & $\begin{array}{c}\text { Muelle } \\
\text { Bariloche }\end{array}$ & $\begin{array}{c}\text { Ortiz } \\
\text { Basualdo }\end{array}$ \\
\hline Brazo Machete & & 0.09 & 0.397 & $0.017^{*}$ & 1 \\
Brazo Blest & 0.09 & & 1 & 1 & 0.1 \\
Club Regatas & 0.397 & 1 & & 1 & 0.434 \\
Muelle Bariloche & $0.017^{*}$ & 1 & 1 & & $0.019^{*}$ \\
Ortiz Basualdo & 1 & 0.1 & 0.434 & $0.019^{*}$ & \\
\hline
\end{tabular}

Tabla 4.Resultado del modelo lineal generalizado testeando el efecto del tamaño del puyen y las características ambientales sobre la comunidad de helmintos de G. maculatus del puyen chico.

Table 4. Results of generalized linear models (GLMs) testing the effects of puyen size and environmental characteristics on the helminth communities of $G$. maculatus of small puyen

\begin{tabular}{lccc}
\hline Predictor & $\begin{array}{c}\text { Probabilidad- } \\
\log \end{array}$ & $\begin{array}{c}\text { Chi } \\
\text { cuadrado }\end{array}$ & $P$ \\
\hline Intersección & -888.883 & & \\
Impacto antrópico & -879.201 & 19.36371 & $6.240567 \mathrm{E}-05$ \\
Vegetación acuática & -875.228 & 7.94730 & $4.815930 \mathrm{E}-03$ \\
Litoral somero & -861.044 & 28.36732 & $1.003452 \mathrm{E}-07$ \\
$\begin{array}{l}\text { Exposición al viento } \\
\text { predominante }\end{array}$ & -861.044 & 0.00000 & $3.000000 \mathrm{E}+30$ \\
Longitud estándar & -841.808 & 38.47164 & $5.555505 \mathrm{E}-10$ \\
\hline
\end{tabular}

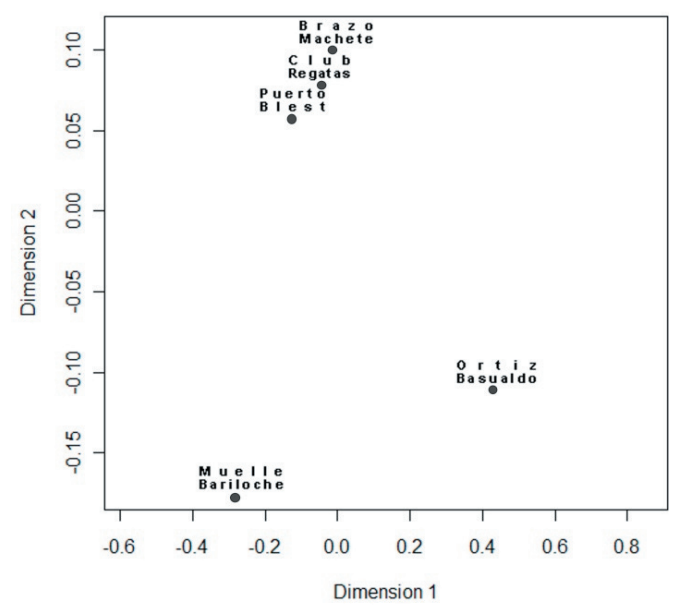

Figura 3. Escalamiento multidimensional (MDS) de los cinco sitios del lago Nahuel Huapi, basados en el índice de Bray Curtis.

Figure 3. Multidimensional scaling (MDS) of five sites in Lake Nahuel Huapi, based on Bray Curtis index.

Tabla 5. Parámetros poblacionales y comunitarios de los parásitos de Galaxias maculatus (intensidad media [IM], prevalencia $[\mathrm{Pr}]$, abundancia $[\mathrm{Ab}]$ y riqueza).

Table 5. Population and community parameters of helminth parasites of Galaxias maculatus (mean intensity [IM], prevalence [Pr], abundance $[\mathrm{Ab}]$ and richness).

\begin{tabular}{|c|c|c|c|c|c|c|c|c|c|c|c|c|c|c|c|}
\hline Sitio & Brazo & $\mathrm{Mac}$ & hete & & erto B & est & & $\overline{b \operatorname{Reg}}$ & $\operatorname{atas}$ & Muel & e Bari & loche & Ortiz & Basu & ldo \\
\hline Helmintos & IM & $\operatorname{Pr}$ & $\mathrm{Ab}$ & IM & $\operatorname{Pr}$ & $\mathrm{Ab}$ & IM & $\operatorname{Pr}$ & $\mathrm{Ab}$ & IM & $\operatorname{Pr}$ & $\mathrm{Ab}$ & IM & $\operatorname{Pr}$ & $\mathrm{Ab}$ \\
\hline Tylodelphys sp. & 98.6 & 80 & 2366 & 84.9 & 96 & 1952 & 81.2 & 90 & 2192 & 36.9 & 93 & 1033 & 231.7 & 50 & 6572 \\
\hline Posthodiplostomum sp. & & & & 1.8 & 21 & 9 & 2 & 13 & 8 & & & & & & \\
\hline Steganoderma szidati & 5.6 & 17 & 28 & 2 & 4 & 2 & & & & 3.5 & 7 & 7 & 2 & 13 & 10 \\
\hline Stephanoprora uruguayense & 2.2 & 33 & 22 & 1.7 & 25 & 10 & 1.1 & 23 & 8 & 1.4 & 17 & 7 & 5.5 & 73 & 120 \\
\hline Acanthostomoides apophalliformis & 1.9 & 47 & 26 & 1 & 21 & 5 & 1.8 & 60 & 33 & 1.3 & 37 & 14 & 2 & 30 & 30 \\
\hline Philureter trigoniopsis & 11.9 & 23 & 83 & & & & 1.6 & 33 & 16 & 3.1 & 30 & 28 & 1.8 & 13 & 20 \\
\hline Gyrodactylus sp. & 1.8 & 20 & 11 & & & & & & & & & & 1 & 3 & 1 \\
\hline Diphyllobothrium sp. & & & & & & & & & & 1 & 3 & 1 & 1 & 3 & 2 \\
\hline Ailinella mirabilis & 1.6 & 27 & 13 & & & & 1 & 13 & 4 & 1.5 & 13 & 6 & & & \\
\hline Acanthocephalus tumescens & 4.2 & 20 & 25 & 1.7 & 13 & 5 & & & & 1 & 7 & 2 & & & \\
\hline Contracaecum sp. & 1 & 13 & 4 & 1 & 13 & 3 & & & & 1 & 3 & 1 & 1 & 3 & 4 \\
\hline Hysterothylacium patagonense & 1.3 & 10 & 4 & 1 & 4 & 1 & 2 & 3 & 2 & 2 & 3 & 2 & 2 & 3 & 2 \\
\hline Placonema pataguense & & & & 1.3 & 13 & 4 & & & & & & & & & \\
\hline Camallanus corderoi & 1 & 3 & 1 & 1 & 8 & 2 & 1 & 7 & 2 & 1 & 3 & 1 & 1 & 3 & 4 \\
\hline Hedruris suttonae & 4.4 & 30 & 40 & 5.3 & 13 & 16 & 2.7 & 10 & 8 & 1 & 3 & 1 & 1.3 & 10 & 6 \\
\hline Riqueza & & 12 & & & 11 & & & 9 & & & 12 & & & 11 & \\
\hline Abundancia total & & 2623 & & & 2009 & & & 2273 & & & 1103 & & & 6771 & \\
\hline
\end{tabular}


Tabla 6. Dominancia de las especies de helmintos en las comunidades componentes de Galaxias maculatus en los cinco sitios del lago Nahuel Huapi. Se indican con asterisco la $1^{\mathrm{a}}$ y la $2^{\mathrm{a}}$ especies dominantes.

Table 6. Dominance of helminth species in component communities of Galaxias maculatus in the five sites of Lake Nahuel Huapi. Asterisks highlight $1^{\text {st }}$ and $2^{\text {nd }}$ dominant species

\begin{tabular}{|c|c|c|c|c|c|}
\hline Sitio & Brazo Machete & Puerto Blest & Club Regatas & Muelle Bariloche & Ortiz Basualdo \\
\hline Tylodelphys sp. & $0.90^{*}$ & $0.98^{*}$ & $0.97^{*}$ & $0.94^{*}$ & $0.99^{*}$ \\
\hline Posthodiplostomum sp. & & 0.005 & 0.004 & & \\
\hline Steganoderma szidati & 0.01 & 0.001 & & 0.006 & 0.001 \\
\hline Diphyllobothrium sp. & & & & 0.001 & 0.0003 \\
\hline Stephanoprora uruguayense & 0.01 & & & & $0.018^{*}$ \\
\hline Acanthostomoides apophalliformis & 0.01 & 0.003 & $0.015^{*}$ & 0.01 & 0.004 \\
\hline Philureter trigoniopsis & $0.032^{*}$ & & 0.01 & $0.03^{*}$ & 0.003 \\
\hline Gyrodactylus sp. & 0.004 & & & & 0.0001 \\
\hline Ailinella mirabilis & 0.005 & & 0.002 & 0.005 & \\
\hline Acanthocephalus tumescens & 0.010 & 0.003 & & 0.002 & \\
\hline Contracaecum sp. & 0.002 & 0.002 & & 0.001 & 0.001 \\
\hline Hysterothylacium patagonense & 0.002 & 0.001 & 0.001 & 0.002 & 0.0003 \\
\hline Placonema pataguense & & 0.002 & & & \\
\hline Camallanus corderoi & 0.0004 & 0.001 & 0.001 & 0.001 & 0.001 \\
\hline Hedruris suttonae & 0.02 & $0.008^{*}$ & 0.004 & 0.001 & 0.001 \\
\hline
\end{tabular}

\section{DisCUSIÓN}

El tamaño del hospedador ha sido reportado como una de las fuentes más notables de variación en la composición de las comunidades de parásitos (Le Pommelet \& Silan 1998). En este sentido, en el lago Nahuel Huapi se observó una tendencia al incremento de la riqueza en los puyenes de mayor tamaño. Los hospedadores con niveles altos en la cadena trófica también generan comunidades de parásitos más ricas y abundantes (Bush et al. 2001; Poulin \& Valtonen 2001; Poulin \& Leung 2011; Garrido-Olvera et al. 2012). El análisis de las comunidades de parásitos de especies de peces de tamaño y de nivel trófico similares a G. maculatus y en escalas geográficas pequeñas muestra rangos de riqueza de helmintos por ambiente de entre 3 y 10 especies para latitudes tropicales como ríos y lagunas de México (Pinacho-Pinacho et al. 2014; Salgado-Maldonado et al. 2014) y de entre 7 y 19 para latitudes mayores (e.g., Canadá y el sudeste de Europa [Dávidová et al. 2011; Chapmann et al. 2015]). En este estudio, las riquezas en G. maculatus se aproximan a los valores obtenidos en altas latitudes. Sin embargo, cuando se consideran las riquezas de este pez presa a escala geográfica regional se registran hasta 33 especies parásitas (Viozzi et al. 2009). Además, un análisis con sólo los ciclos heteroxenos (i.e., los que requieren interacción trófica entre los hospedadores) muestra que los peces presa con tallas y nivel trófico similares a los de G. maculatus actúan principalmente como hospedadores intermediarios ya que presentan una proporción de especies larvales de más de $60 \%$ en las comunidades componentes (Dávidová et al. 2011; Pinacho-Pinacho et al. 2014; Salgado-Maldonado et al. 2014; Chapmann et al. 2015). Esta proporción es mayor que la de los peces piscívoros y de mayor tamaño que suelen actuar como hospedadores definitivos y presentan una proporción de larvas por lo general menor al 30\% (Poulin \& Valtonen 2002; Morozińska-Gogol 2013).

La composición y la abundancia de las especies de las comunidades de helmintos de G. maculatus en el lago Nahuel Huapi permite separar los sitios localizados en el oeste del lago (Brazo Machete, Club Regatas y Puerto Blest) de aquellos ubicados más hacia el este, sobre su cuerpo principal (Muelle Bariloche y Ortiz Basualdo). Este lago abarca distintos ecosistemas terrestres que van desde la selva valdiviana en el oeste a la estepa patagónica en el este, a lo que se suman diferencias en el relieve de sus costas. Este gradiente contribuiría a la distribución diferencial de los hospedadores definitivos, que en el caso de los ciclos alogénicos adquiere particular relevancia dado que requieren aves o mamíferos como hospedadores definitivos. La distribución de aves ictiófagas como Larus dominicanus (gaviotas) y Phalacrocorax brasilianus (cormoranes) podrían generar similitudes entre los sitios Ortiz Basualdo y Muelle Bariloche, ubicados en el cuerpo principal del lago (mitad este). Estos dos sitios comparten la presencia del cestode 
Diphyllobothrium sp., que parasita a humanos y a gaviotas (Semenas \& Kreiter 1995; Kreiter \& Semenas 1997; Casalins et al. 2015). Si bien los sitios de anidación de gaviotas registrados hasta el momento se encuentran cercanos a la isla Victoria (Frixione et al. 2012), se observan sitios de descanso y/o alimentación en el sector este del lago, donde se registra un alto impacto antrópico en la costa sudeste, sobre todo en las desembocaduras de ríos como el Nireco y Nirihuau (Figura 1), probablemente debido a su cercanía al matadero, a ahumaderos, al vertedero municipal y a la zona de eliminación de desechos cloacales. En avistajes cuantitativos de aves, la abundancia de $P$. brasilianus resulta mayor en lugares próximos a la ciudad de Bariloche que en otros sitios (Veleizán 2009). Ambos sitos se diferencian entre sí por la altas abundancias de Tylodelphys sp. y de S. uruguayense en el sitio Ortiz Basualdo. Estas larvas de digeneos con ciclos alogénicos también usan aves como hospedadores definitivos.

La presencia o ausencia de macrófitas es importante en los ciclos de los parásitos dado que brinda diferentes condiciones para el asentamiento de hospedadores intermediarios y definitivos. Club Regatas y Puerto Blest, que presentan mayor abundancia de juncos, son semejantes en la composición y abundancia de helmintos y los únicos donde se registró infección por Posthodiplostomum sp., digeneo diplostómido que utiliza al caracol Anysancilus obliquus como primer hospedador intermediario, que vive generalmente adherido a juncos (Ritossa et al. 2014). En lagos sin macrófitas, donde los puyenes no tienen refugio, los valores de infección del monogeneo Philureter trigoniopsis son significativamente más altos que en aquellos cuyas costas presentan juncales (Viozzi \& Semenas 2009). En concordancia con estos autores, en este estudio los sitios con mayor abundancia de macrófitas (Puerto Blest y Club Regatas) fueron aquellos en donde $P$. trigoniopsis presentó los valores más bajos de intensidad media y prevalencia, o estuvo ausente.

Se ha reportado flujo génico entre las diferentes poblaciones de G. maculatus de este lago; podría deberse a que las corrientes de la zona limnética pueden transportar a las larvas de puyenes a otras zonas litorales de las cuales no son originarias (Carrea 2011; Carrea et al. 2012). Considerando que en estadios larvales premetamórficos y metamórficos de G. maculatus capturados en la zona limnética del lago Moreno (de características similares y ubicado en el mismo Parque Nacional) no se encontraron parásitos (Viozzi \& Semenas 2009), se puede deducir que estas larvas, que consumen sólo algas y larvas naupli de ciclopoideos (Cervellini et al. 1993), no contribuirían a la dispersión de parásitos entre los diferentes sitios del lago. Otra explicación alternativa podría ser que los adultos migran entre sitios. Sin embargo, las diferencias encontradas entre las comunidades componentes de helmintos de G. maculatus apoyan la hipótesis de una baja tasa de migración de los peces juveniles $\mathrm{y}$ adultos entre los sitios.

Las diferencias ambientales en las costas de los sitios muestreados propician variaciones entre las comunidades de helmintos. Sin embargo, las comunidades componentes de los cinco sitios están dominadas por Tylodelphys sp., lo que genera cierta homogeneidad entre ellas. Este digeneo utiliza caracoles del género Chilina, que domina las comunidades macrobentónicas no sólo del lago Nahuel Huapi (Veleizán 2009) sino, en general, de todos los lagos de la región andino-patagónica (Flores 2005). Esta ubicuidad del hospedador intermediario le confiere a esta especie de digeneo una capacidad elevada de colonización, que permite que esté ampliamente distribuida en los ambientes patagónicos (Viozzi et al. 2009). Tylodelphys sp. presentó una menor abundancia y una intensidad media en el sitio Muelle Bariloche. Si bien el viento no tuvo efecto sobre la composición y la distribución de las abundancias de las especies de helmintos, podría ser responsable de las bajas abundancias de Tylodelphys sp. en el Muelle Bariloche ya que de todos los sitios estudiados, éste es el único que presenta una alta y casi constante influencia del viento predominante del oeste-noroeste. Este efecto provoca un movimiento prácticamente continuo en el agua que podría dificultar la infección activa de las larvas nadadoras como los miracidios y las cercarias a los hospedadores del ciclo, generando así una menor abundancia e intensidad media en G. maculatus.

Aunque todas las comunidades componentes estuvieron dominadas por la misma especie, este trabajo muestra que factores del hospedador (como la longitud del pez) y del ambiente (como la presencia de litorales someros amplios con vegetación acuática 
emergente y el impacto antrópico) generan diferencias en la composición y la abundancia de las especies en las infracomunidades y comunidades componentes de helmintos de G. maculatus en diferentes sitios de un mismo lago.

\section{BiBLIOGRAFÍA}

ANDERSOn, TK \& MVKSukdheo. 2009. Abiotic versus biotic hierarchies in the assembly of parasite populations. Parasitology, 137:743-754.

Barriga, JP; MA Battini; PJ Macchi; D Milano \& VE Cussac. 2002. Spatial and temporal distribution of landlocked Galaxias maculatus and Galaxias platei (Pisces: Galaxiidae) in a lake in the South American Andes. Mar. Fresh. Res., 36:345-359.

Barriga, JP; MA Battini; M García Asorey; C Carrea; PJ Macchi; et al. 2012. Intraspecific variation in diet, growth, and morphology of landlocked Galaxias maculatus during its larval period: the role of food availability and predation risk. Hydrobiologia, 679:27-41.

Bush, AO; K Lafferty; J Lotz \& A Shostak. 1997. Parasitology meets ecology on its own terms: Margolis et al. revisited. J. Parasitol., 83:575-583.

Bush, AO; JC Fernández; GW Esch \& R Seed. 2001. Parasitism: The diversity and ecology of animal parasites. Cambridge University Press. Cambridge, UK. Pp. 565.

Carrea, C. 2011. Ecología, genética e historia de vida de Galaxias maculatus en dos grandes cuencas de Patagonia. Tesis Doctoral. Universidad Nacional del Comahue. San Carlos de Bariloche, Argentina. Pp. 169.

Carrea, C; JP Barriga; VE Cussac \& DE Ruzzante. 2012. Genetic and phenotypic differentiation among Galaxias maculatus populations in a Patagonian postglacial lake system. Biol. J. Linn Soc., 107:368-382.

Casalins, L; M Arbetman; L Semenas; A Veleizán; V Flores; et al. 2015. Difilobotriosis en gaviotas. Pasado y presente de esta zoonosis en el Parque Nacional Nahuel Huapi. Rev. Arg. Zoon. Enf. Infec. Emerg., 10:38-39.

Cervellini, P; M Battini \& V Cussac. 1993. Ontogenetic shifts in the diet of Galaxias maculatus (Galaxiidae) and Odontesthes microlepidotus (Atherinidae). Environ. Biol. Fish., 36:283-290.

Chapman, J; D Marcogliese; C Suski \& S Cooke. 2015. Variation in parasite communities and health indices of juvenile Lepomis gibbosus across a gradient of watershed land-use and habitat quality. Ecol. Ind., 57:564-572.

Cussac, V; P Cervellini \& M Battini. 1992. Intralacustrine movements of Galaxias maculatus (Galaxiidae) and Odontesthes microlepidotus (Atherinidae) during their early life history. Environ. Biol. Fish., 35:141-148.

Dávidová, M; R Blažek; T Trichkova; E Koutrakis; Ö Gaygusuz; et al. 2011. The rol of the European bitterling (Rhodeus amarus, Cyprinidae) in parasite accumulation and transmission in riverine ecosystems. Aquat. Ecol., 45:377-387.

Esch, G; A Shostak; D Marcogliese \& T Goater. 1990. Patterns and processes in helminth parasite communities: an overview. Capítulo 1. Pp. 1-19 en: Esch, G; A Bush \& J Aho (eds.). Parasite Communities: patterns and processes. Chapman \& Hall. Londres, UK. Pp. 335.

Fellis, KJ \& GW Esch. 2005. Autogenic-allogenic status affects interpond community similarity and species area relationship of macroparasites in the bluegill sunfish,
Lepomis macrochirus, from a series of freshwater ponds in the Piedmont area of North Carolina. J. Parasitol., 91: 764-767.

Fernández, MV; NL Brugni; GP Viozzi \& L Semenas. 2010. The relationship between fish assemblages and the helminth communities of a prey fish, in a group of small shallow lakes. J. Parasitol., 96:1066-1071.

Flores, V. 2005. Estructura comunitaria de digeneos larvales en Chilina dombeiana y Heleobia hatcheri (Mollusca, Gastropoda) de la región andino patagónica. Tesis Doctoral. Universidad Nacional del Comahue. San Carlos de Bariloche. Pp. 346.

Frixione, M; R Casaux; C Villanueva \& P Alarcón. 2012. A recently established Kelp Gull colony in a fresh water environment supported by an inland refuse dump in Patagonia. Emu, 112:174-178.

Garrido-Olvera, L; HT Arita \& G Pérez-Ponce De León. 2012. The influence of host ecology and biogeography on the helminth species richness of freshwater fishes in Mexico, Parasitology, 139:1652-665.

Hartvigsen, R \& CR Kennedy. 1993. Patterns in the composition and richness of helminth communities in brown trout, Salmo trutta, in a group of reservoirs. J. Fish Biol., 43:603-615.

Karvonen, A \& T Valtonen. 2004. Helminth assemblages of whitefish (Coregonus lavaretus) in interconnected lakes: similarity as a function of species specific parasites and geographical separation. J. Parasitol., 90:471-476.

Kreiter, A \& L Semenas. 1997. Helmintos parásitos de Larus dominicanus en la Patagonia Argentina. Bol. Chil. Parasitol., 52:39-42.

Lafferty, K. 2008. Effects of diseases on community interactions and foods web structure. Pp. 205-222 en: Ostfeld, R; F Keesing \& V Eviner (eds.). Infectious Disease Ecology. Princeton University Press, Princeton, Estados Unidos. Pp. 506.

Le Pommelet, E \& P Silan. 1998. Gut of goatfishes, a heterogeneous biotope for intestinal mesoparasites: variations in pyloric caeca number and growth models of colonizable digestive surface area. J. Fish. Biol., 53:866-878.

Macchi, P. 2004. Respuestas de Galaxias maculatus a la depredación por parte de Percichthys trucha y los salmónidos introducidos en ambientes lénticos de la Patagonia norte. Tesis Doctoral, Universidad Nacional del Comahue, San Carlos de Bariloche, Argentina. Pp. 175.

Morozińska-Gogol, J. 2013. Parasite communities of European perch, Perca fluviatilis L. (Actinopterygii: Perciformes: Percidae) from lake Łebsko (Central Coast, Poland). Ann. Parasitol., 59:89-98.

Pedrozo, F \& P Vigliano. 1995. Lago Nahuel Huapi. En: Catálogo de lagos y embalses de la Argentina. AMR y asociados. Buenos Aires, Argentina. Pp. 328.

Pedrozo, F; R Alcalde \& M Manuel. 1997. Estado trófico del lago Nahuel Huapi y estimación preliminar de su posible evolución. CRUB- DPA, San Carlos de Bariloche, Argentina. Pp. 48.

Pinacho-Pinacho, C; MA Pérez-Ruíz; AL Sereno-Uribe; M García-Varela \& E Martínez-Ramírez. 2014. Richness and similarity of helminth communities of the freswater fish Profundulus punctatus (Pisces: Cyprinodontidae) from Oaxaca, México. Rev. Mex. Biodiv., 85:1129-1138.

Poulin, R \& ET Valtonen. 2001. Nested assemblages resulting from host size variation: the case of endoparasite communities in fish hosts. Int. J. Parasitol., 31:1194-1204. 
Poulin, R \& ET Valtonen. 2002. The predictability of helminth community structure in space: a comparison of fish populations from adjacent lakes. Int. J. Parasitol., 32:1235-1243.

Poulin, R \& TLF Leung. 2011. Latitudinal gradient in the taxonomic composition of parasite communities. J. Helminthol., 85:228-233.

Quirós, R. 1990. Predictors of relative fish biomass in lakes and reservoirs of Argentina. Can. J. Fish. Aquat. Sci., 47:928-939.

Rechencq, M; A Sosnovsky; PJ Macchi; PA Alvear \& PH Vigliano. 2011. Extensive diel fish migrations in a deep ultraoligotrophic lake of Patagonia Argentina. Hydrobiol., 658:147-161.

Ritossa, G; V Flores \& G Viozzi. 2014. Infection dynamics of Posthodiplostomum sp. (Digenea: Diplostomiadae) in first and second intermediate hosts from an Andean Patagonian lake. Rev. Arg. Parasitol., 3:16-23.

Rodhe, K. 2010. Marine parasite diversity and environmental gradients. Cap 6. en: Morand, S \& B Krasnov (eds.). The biography of Host-Parasite interactions. Oxford University Press, Oxford, Reino Unido. Pp. 277.

Salgado-Maldonado, G \& CR Kennedy. 1997. Richness and similarity of helminth communities in the tropical cichlid fish Cichlasoma urophthalmus from the Yucatan Peninsula, Mexico. Parasitology, 114:581-590.

Salgado-Maldonado, G; R Aguilar-Aguilar; G CabañasCarranza; E Soto-Galera \& C Mendoza-Palmero. 2005. Helminth parasite in freshwater fish from the Papaloapan river basin, Mexico. Parasitol. Res., 96:69-89.

Salgado-Maldonado, G; MT Novello-Turcotte; G Vázquez; JM Caspeta-Mandujano; B Quiróz-Martínez; et al. 2014. The communities of helminth parasites of Heterandria bimaculata (Teleostei: Poeciliidae) from the upper Río La
Antigua basin, east-central México show a predictable structure. Parasitology, 141:970-980.

Semenas, L \& A Kreiter. 1995. Epidemiología de la difilobotriasis en la Región Andino Patagónica. Rev. Asoc. Bioq. Arg., 59:203-206.

Ternengo, S; C Levron; D Mouillot \& B Marchand. 2009. Site influence in parasite distribution from fishes of the Bonifacio Strait Marine Reserve (Corsica Island, Mediterranean Sea). Parasitol. Res., 104:1279-1287.

Timi, J; AL Lanfranchi \& JL Luque. 2010. Similarity in parasite communities of the teleost fish Pinguipes brasilianus in the southwestern Atlantic: Infracommunities as a tool to detect geographical patterns. Int. J. Parasitol., 40:243-254.

Veleizán, A. 2009. Efecto de la heterogeneidad ambiental sobre la comunidad de digeneos larvales de Chilina spp. (Gastropoda) en lagos de la Patagonia Argentina. Tesis para optar al grado de Licenciado en Ciencias Biológicas. Universidad Nacional del Comahue, San Carlos de Bariloche, Argentina. Pp. 66.

Vidal-Martínez Vm \& R Poulin. 2003. Spatial and temporal repeatability in parasite community structure of tropical fish hosts. Parasitology, 127:387-398.

Viozzi, G \& L Semenas. 2009. Do environmental differences between lakes in northwestern Argentinean Patagonia affect the infection of Philureter trigoniopsis (Monogenea) in Galaxias maculatus (Osmeriformes)? J. Parasitol., 95:25-31.

Viozzi, G; L Semenas; N Brugni \& V Flores. 2009. Metazoan parasites of Galaxias maculatus (Osmeriformes: Galaxiidae) from Argentinean Patagonia. Comp. Parasitol., 76:229-239.

Winiewski,WL.1958. Characterization of the parasitofauna of an eutrophic lake. Acta Parasitol. Polon., 6:1-64. 